\title{
Editorial: Ecological, Behavioral and Genomic Consequences in the Rodent Family Sciuridae: Why Are Squirrels So Diverse?
}

\author{
Jane M. Waterman ${ }^{1}$, Toni I. Gossmann ${ }^{2 \star}$, Oleg Brandler ${ }^{3}$ and John L. Koprowski ${ }^{4}$ \\ ${ }^{1}$ Department of Biological Sciences, University of Manitoba, Winnipeg, MB, Canada, ${ }^{2}$ Department of Animal Behaviour, \\ Bielefeld University, Bielefeld, Germany, ${ }^{3}$ Koltzov Institute of Developmental Biology, Russian Academy of Sciences, \\ Moscow, Russia, ${ }^{4}$ Haub School of Environment and Natural Resources, Laramie, WY, United States
}

Keywords: speciation, hybridization, keystone species, adaptation, climate change, wild species, marmots

\section{Editorial on the Research Topic}

Ecological, Behavioral and Genomic Consequences in the Rodent Family Sciuridae: Why Are Squirrels So Diverse?

Rodents form the most diversified mammalian clade, but our knowledge about this intriguing animal group is centered around a few taxa, particularly the Muridae. However, another exceptional example of rodent diversification is the family Sciuridae (squirrels), the most speciose family after Muridae (murids). With $>300$ species living across the globe in a range of habitats such as deserts, high altitudes and rainforests, their diversity in morphology, ecology, and behavior is broad. Squirrels' lifestyles are extremely diverse - there are climbing, flying, and burrowing forms. Distinct species within a single genus can live in extremely different conditions, such as marmots or ground squirrels that range from arid to permafrost regions. Along with their outstanding diversity, sciurids play an important role in the formation and function of ecosystems. Many are considered keystone species and ecosystem engineers for their ability to influence the environment and create new habitat niches. Despite their importance, many populations have already experienced severe fragmentation and decline, mainly due to habitat loss, overhunting, and persecution as pests. Some squirrel species, however, are successfully surviving and even thriving in anthropogenic biotopes.

The underlying mechanisms and consequences of the diversification of squirrels remain poorly understood. To understand the selective forces influencing their evolution, it is crucial to broadly examine their behavioral, ecological, and morphological adaptations. As many members of the Sciuridae possess extreme features in relation to sociality, behavior, metabolism, and ecosystems, they are, in fact, model species for the functional and evolutionary underpinnings of unusual and extreme traits. This Research Topic explores the link between ecosystem adaptation and species diversification, in particular for squirrels that have gained little attention in the literature thus far.

Ecologically, squirrels live in habitats that vary in altitude, latitude, and extremes of environmental conditions from arid-adapted to arctic-adapted. For many species, resource availability is a driver for social systems and home range dynamics, and differences in the timing of these resources suggest that extrinsic factors rather than body size, influenced home range, and territoriality in two closely related tree squirrels (Mazzamuto et al.). Changes in habitat, particularly changes caused by human impacts, can lead to changes in species interactions, as Sovie et al. found using occupancy models to examine habitat use in North American and European red squirrels. Local adaptation to food resources is a form of ecological plasticity that may help species to cope with rapid habitat changes, such as anthropogenic transformation, as demonstrated for 
steppe marmots inhabiting the dry grasslands of Eurasia (Ronkin et al.). The high food selectivity of the European subspecies of the steppe marmot makes it very vulnerable to degradation of these grasslands with the cessation of grazing cattle. In contrast, the Kazakh subspecies has lower dietary selectivity and therefore allows it to respond more successfully to anthropogenic changes of grasslands.

While habitat change impacts steppe marmots, in North America, the opposite pattern of squirrels affecting habitat is seen in a study of the ecosystem engineering black-tailed prairie dog. This species is well-known as a keystone species in many prairie grasslands, but Hale et al. demonstrate they can also restore ecosystem functions, by limiting the expansion and proliferation of woody plants. Such tight-knit interactions between squirrels and their habitats are also reflected in a paper focusing on squirrels as predators and seed dispersers (Steele and Yi). While squirrels can negatively impact the seeds of many tree species, their scatter-hoarding behaviors are also critical in the dispersal of many trees and many tree species have co-evolved with squirrels in areas of high squirrel density to reduce the costs of squirrel predation.

For almost a decade the available genomic resources for squirrel species were limited to the thirteen-lined ground squirrel genome. However, recently several marmot genomes have been published (Alioto et al., 2019; Bai et al., 2019; Gossmann et al., 2019; Puiu et al., 2020). The study by Junker and Gossmann uses the available genomic resources of marmots to study the molecular signatures of a key aging protein family, Sirtuins. They find that SIRT1, a key player in human aging research, shows a molecular signature of adaptation which they argue might be linked to the extreme lifestyle of marmot species. The relevance of the genomic resources also becomes apparent in a natural hybrid zone of two marmot species, Marmota baibacina and $M$. sibirica (Brandler et al.). Although the study has only a limited marker number, it is the first evidence of hybridization of marmot species in the wild. Understanding the genomic underpinnings of natural hybridization is a major task in evolutionary and conservation biology and is particularly important for squirrels because of their role as keystone species. While hybridization is a key mechanism of increasing genetic complexity, it is predated by how species diverge. Two studies look at particular examples in squirrels. First, the study by Fiedler de Abreu-Jr et al. looks at diversification in South American tree squirrels, a potential textbook example of rapid species diversification. They find that the South American invasion was not as recent as previously inferred, but that the species diversification there was indeed quite fast and impactful. Second, Hinckley et al. investigate species diversification in Sundaland squirrels and identify allopatry as the main driver of diversification but also show that ecomorphological and behavioral adaptations were important and suggest this diversification as evidence for niche divergence. The diversifications in South American and Sundaland squirrels as well as hybridization in a secondary contact zone and adaptive extension of lifespan in marmots illustrate responses to global paleoclimatic changes. They are powerful examples that indicate the potential of squirrels to adapt and thereby not only maintain but also increase their species richness.

The need for clade-wide genome sequencing of squirrel species is not only fostering our knowledge on how this remarkable rodent family diversified but could also shed light on how important traits have contributed to it. The study of Sheets and Chavez investigates whether Gloger's rule, the prediction that darker colored animals are associated with environments that are warmer and more moist, is supported among squirrel species. Based on data from 137 species using phylogenetic and univariate regression methods they find that the rule generally applies. Identifying the genomic regions underlying Gloger's rule using genomic approaches would be the natural next step. However, establishing the molecular underpinnings of trait diversification on macroevolutionary scales is only feasible when large-scale genomic and phenotypic data are available (Yusuf et al., 2020). There is also an urgent need to complement large scale comparative studies with microevolutionary approaches, such as the study by Potash et al.. They investigate, using a nongenomic approach, pelage polymorphism in Easter fox squirrels and its relation to environmental heterogeneity-ultimately concluding that anthropomorphic influences will likely lead to a loss of pelage diversity. Newar and Bowman examine another important trait in their study-vocalization-and review how vocalization has been studied in squirrels and investigate possible explanatory variables of the evolution of vocalization. Indeed they find that open habitat and social complexity are associated with high frequency sounds, but body mass is associated with low frequencies.

A key question in evolutionary biology considers whether diversification of particular traits is associated with fitness benefits. Showing this in empirical data is often difficult, because many confounding factors may obscure a signal of fitness advantage. The study by Dobson et al. investigates different measures in Columbian ground squirrels and highlights that population dynamics and specific features such as longevity have to be accounted for when estimating fitness.

The diversity and breadth of adaptations in the Family Sciuridae support this group's potential to gain insight into our natural world. These large rodents (relative to the size of most rodents) that are primarily diurnal, may allow us to address genetic, behavioral, and ecological questions that are more difficult in their smaller more cryptic cousins. The studies in this special topic point the way to new questions and highlight the potential of this group as excellent models for studies in evolutionary biology and ecology.

\section{AUTHOR CONTRIBUTIONS}

JW and TG drafted the manuscript. All authors contributed to revising and finalizing the manuscript.

\section{FUNDING}

OB was funded by IDB RAS State Assignment for Basic Research 0088-20210019. 


\section{REFERENCES}

Alioto, T. S., Cruz, F., Gómez-Garrido, J., Triyatni, M., Gut, M., Frias, L., et al. (2019). The genome sequence of the eastern woodchuck () A preclinical animal model for chronic hepatitis B. G3 9, 3943-3952. doi: $10.1534 / \mathrm{g} 3.119 .400413$

Bai, L., Liu, B., Ji, C., Zhao, S., Liu, S., Wang, R., et al. (2019). Hypoxic and cold adaptation insights from the himalayan marmot genome. iScience 11, 505-507. doi: 10.1016/j.isci.2019.01.019

Gossmann, T. I., Shanmugasundram, A., Börno, S., Duvaux, L., Lemaire, C., Kuhl, H., et al. (2019). Ice-age climate adaptations trap the alpine marmot in a state of low genetic diversity. Curr. Biol. 29, 1712-1720.e7. doi: 10.1016/j.cub.2019.04.020

Puiu, D., Zimin, A., Shumate, A., Ge, Y., Qiu, J., Bhaskaran, M., et al. (2020). The genome of the American groundhog. F1000Res. 9:1137. doi: 10.12688/f1000research.25970.1

Yusuf, L., Heatley, M. C., Palmer, J. P. G., Barton, H. J., Cooney, C. R., and Gossmann, T. I. (2020). Noncoding regions underpin avian bill shape diversification at macroevolutionary scales. Genome Res. 30, 553-565. doi: $10.1101 /$ gr.255752.119
Conflict of Interest: The authors declare that the research was conducted in the absence of any commercial or financial relationships that could be construed as a potential conflict of interest.

Publisher's Note: All claims expressed in this article are solely those of the authors and do not necessarily represent those of their affiliated organizations, or those of the publisher, the editors and the reviewers. Any product that may be evaluated in this article, or claim that may be made by its manufacturer, is not guaranteed or endorsed by the publisher.

Copyright (c) 2021 Waterman, Gossmann, Brandler and Koprowski. This is an open-access article distributed under the terms of the Creative Commons Attribution License (CC BY). The use, distribution or reproduction in other forums is permitted, provided the original author(s) and the copyright owner(s) are credited and that the original publication in this journal is cited, in accordance with accepted academic practice. No use, distribution or reproduction is permitted which does not comply with these terms. 